\title{
Differences in HLA-B27 positive and negative patients with ankylosing spondylitis: study of clinical disease activity and concentrations of serum $\operatorname{Ig} \mathrm{A}, \mathrm{C}$ reactive protein, and haptoglobin*
}

Case Western Reserve
University,
Department of Medicine,
Division of
Rheumatology,
MetroHealth Medical
Centre, Cleveland,
OH, USA and
Division of
Rheumatology,
University of Limburg,
Maastricht,
The Netherlands
T L Reynolds
M A Khan
S van der Linden
R P Cleveland
Correspondence to:
Dr M A Khan,
Division of Rheumatology,
MetroHealth Medical Centre,
3395 Scranton Road,
Cleveland, OH 44109 , USA.
Accepted for publication
22 February 1990

*This work was presented at the 1989 annual meeting of the American College of Rheumatology.

\begin{abstract}
To find out whether disease activity and B27 status were associated with serum concentrations of IgA, C reactive protein (CRP), and haptoglobin in ankylosing spondylitis (AS) multivariate analysis of variance was used to study 101 patients with AS whose disease was clinically classified as active or inactive, and who were HLA-B27 typed. It was found that B27 and disease activity do interact significantly to affect the serum concentrations of IgA, CRP, and haptoglobin. When the 77 B27+ patients were examined, however, it was found that disease activity was significantly associated with serum concentrations of CRP and haptoglobin, but not with serum IgA. In contrast, in the 24 B27 - patients concentrations of serum IgA were significantly associated with disease activity, but concentrations of $\mathbf{C R P}$ and haptoglobin were not. These results emphasise the known difference between B27+ and B27- AS and suggest different pathogenic mechanisms in the two forms of AS.
\end{abstract}

Ankylosing spondylitis (AS) shows a remarkable association with HLA-B27, and mounting evidence suggests that the B27 gene may have a pathogenic role in the development of $\mathrm{AS}^{1-3}$ Several investigators have also noted that serum concentrations of IgA and certain serum markers of inflammation, such as $\mathrm{C}$ reactive protein (CRP) and haptoglobin, are raised in patients with AS. ${ }^{4-6}$

We performed this study to investigate pathophysiological differences between $\mathrm{B} 27+$ and B27 - patients with AS and relate these differences to the serum concentrations of IgA, CRP, and haptoglobin to determine whether the B27 status and presence or absence of disease activity could predict the concentrations of $\operatorname{IgA}$, CRP, and haptoglobin.

\section{Patients and methods \\ PATIENTS}

We studied 303 unrelated white Swiss patients with definite AS as defined by the New York criteria $^{7}$ and by the modified criteria. ${ }^{8}$ In addition, each patient's disease activity was clinically classified as either active or inactive by one of us (SVL) according to the clinical criteria proposed by Sheehan et al. ${ }^{9}$ Active disease was defined as either $(a)$ an exacerbation of spinal pain/spinal stiffness (which had usually required an increase in anti-inflammatory drugs within the previous four weeks) or (b) one of the following: active peripheral arthritis, acute anterior uveitis, or a combination of a positive response to sacroiliac stress tests plus a minor extraspinal feature-for example, Achilles tendinitis, plantar fasciitis.

All these patients were HLA-typed. The patients were categorised by presence or absence of HLA-B27, and active or inactive disease. Random samples were chosen from these patients as follows: 31 patients with B27+ inactive disease, 46 patients with $\mathrm{B} 27+$ active disease, six patients with B27- inactive disease, and 18 patients with B27- active disease. Serum samples from these 101 patients were assayed for serum IgA, CRP, and haptoglobin.

\section{METHODS}

HLA typing was performed by the standard microlymphocytotoxicity assay. Serum concentrations of $\operatorname{IgA}$ and CRP were determined by radial immunodiffusion. ${ }^{10}$ Serum concentrations of haptoglobin were measured by electroimmunoassay ${ }^{11}$ using human serum calibrator kit (Atlantic Antibodies, Boston, MA).

\section{STATISTICS}

Statistical results were calculated using 'SPSS/ PC+' (SPSS Inc, Chicago, Illinois). Mean serum values of IgA, CRP, and haptoglobin were calculated for each of the four groups: $\mathrm{B} 27+$ inactive AS, B27+ active AS, B27inactive AS, and B27- active AS. As we have previously shown that serum CRP and haptoglobin correlate statistically with concentrations of serum IgA in patients with $\mathrm{AS},{ }^{4}$ the concentrations of these proteins cannot necessarily be considered to be the result of independent physiological events and hence must be studied together. Thus we used multivariate analysis of variance (MANOVA), a statistical test that takes into account potential interactions between physiological variables which are declared as dependent. These three serum proteins (IgA, CRP, and haptoglobin) were thus declared as the dependent variables. Once an overall trend was established using MANOVA, univariate $F$ statistics were used to determine the contribution of each dependent variable to any noted group difference.

In our first test B27 status and disease activity were declared as independent variables to see if they had a group effect on serum concentrations 
Mean concentrations (mg/l) of serum IgA, C reactive protein (CRP), and haptoglobin in 101 patients with ankylosing spondylitis $(A S)$ grouped by $B 27$ and disease activity status. Values are given as means (SD)

\begin{tabular}{llrl}
\hline Group & Serum IgA & Serum CRP & Serum haptoglobin \\
\hline B27+ Inactive AS $(\mathbf{n}=31)$ & $2660(930)$ & $3 \cdot 2(4 \cdot 8)$ & $1334(493)$ \\
B27+ Active AS $(\mathrm{n}=46)$ & $2850(1542)$ & $9 \cdot 7(14 \cdot 1)$ & $1682(655)$ \\
B27- Inactive AS $(\mathbf{n}=6)$ & $4665(2748)$ & $13 \cdot 6(24 \cdot 0)$ & $1731(649)$ \\
B27- Active AS $(\mathbf{n}=18)$ & $2316(1232)$ & $4 \cdot 0(7 \cdot 6)$ & $1168(606)$ \\
\hline
\end{tabular}

of IgA, CRP, and haptoglobin. Secondly, we analysed only B27+ patients, declaring activity status as the sole independent variable to see which serum proteins were significantly affected. Finally, we selected only B27patients, again declaring disease activity as the independent variable, to examine the effects on serum IgA, CRP, and haptoglobin in B27patients.

\section{Results}

The table shows mean serum concentrations of IgA, CRP, and haptoglobin in the following four groups: B27+ inactive AS, B27+ active AS, B27- inactive AS, and B27- active AS. Subsequently, MANOVA was used to study the interaction between B27 and disease activity in influencing concentrations of the three serum proteins: IgA, CRP, and haptoglobin. When B27 and disease activity were established as independent variables they were shown to interact significantly as a whole to affect the concentrations of $\operatorname{IgA}, \mathrm{CRP}$, and haptoglobin $(p<0.003$, Hotellings F statistic $=0 \cdot 157, n=101)$.

To understand better the nature of this interaction the 77 B27+ patients were analysed using disease activity as the sole independent variable. We then performed MANOVA and looked at the individual univariate $F$ statistics to see whether each protein was affected equally by disease activity. In these B27+ patients disease activity was significantly associated with both serum CRP ( $p<0.017$, univariate F statistic $=5.93, n=77$ ) and serum haptoglobin $(p<0.014$, univariate $F$ statistic $=6.32, n=77)$, but was not significantly associated with serum IgA concentrations.

In contrast, when the 24 B27- patients were studied and disease activity was established as an independent variable only concentrations of serum IgA were significantly associated with disease activity status $(\mathrm{p}<0.008$, univariate $F$ statistic $=8 \cdot 60, n=24)$; neither serum CRP nor serum haptoglobin were significantly associated with disease activity in B27- patients.

\section{Discussion}

Research into the pathophysiological mechanisms of AS is problematic because of the presumed heterogeneous nature of the disease. ${ }^{12} 13$ Although a genetic association with HLA-B27 is clearly established, studies which attempt to correlate laboratory measurements in AS with disease activity have been criticised because of the lack of an accepted standard for distinguishing between active and inactive AS. Investigators have long sought objective criteria for assessing disease activity, including radiological changes, symptomatic reporting, changes in symptoms when anti-inflammatory drugs are given, CRP or erythrocyte sedimentation rate (ESR) values, or both, peripheral joint disease, iritis, and a positive sacroiliac test. As Sheehan's research indicates, no disease activity assessment standard has been accepted to date. ${ }^{9}$

The previous studies demanded a rigorous analysis of disease activity because many of them attempted to determine clinically relevant thresholds for serum proteins that could be used as serum markers of inflammation and thus document flare ups of inflammatory activity in a patient. In contrast, our study, which is cross sectionally designed, attempted to examine the pathogenesis of AS. We sought to separate active from inactive AS in order to explore trends in serum IgA, CRP, and haptoglobin in clinical groups that are more homogeneous than just the presence or absence of AS. We were interested in examining the changes in serum IgA, CRP, and haptoglobin in AS that is clinically active versus currently inactive; our aim was to explore how concentrations of these three proteins might relate to the presence or absence of B27. Indeed, as the table indicates, there is considerable overlap between concentrations of proteins in each of the four groups. We attempted to show a statistical trend and not to find a threshold value for one of the proteins that was both sensitive and specific. Hence our criteria for disease activity relied heavily on symptomatic reporting and essentially considered active disease as a flare up of spinal disease or peripheral arthritis.

The current research represents an extension of our previous efforts. We have previously shown that concentrations of serum IgA and six acute phase proteins (CRP, haptoglobin, $\alpha_{1}$ antitrypsin, $\alpha_{1}$ antichymotrypsin, caeruloplasmin, and $\alpha_{1}$ glycoprotein) were significantly raised in patients with AS compared with healthy controls. ${ }^{4}$ Moreover, we had found that in patients with AS concentrations of serum $\operatorname{IgA}$ correlated significantly with concentrations of four of these acute phase proteins: CRP, haptoglobin, $\alpha_{1}$ antitrypsin, and $\alpha_{1}$ glycoprotein. Several investigators have suggested that gastrointestinal immunostimulation triggers AS in genetically susceptible subjects, ${ }^{4-614-16}$ and that the rise in serum IgA so commonly found in this disease may be a reflection of enhanced production of IgA by gut mucosa. ${ }^{5}$ Moreover, enteric mucosa in patients with AS shows histological changes on biopsy which are consistent with possible impairment of the mucosal barrier that might allow entry of gut derived antigens past the gut epithelium. ${ }^{16} \mathrm{We}$ suggested that if markers of gastrointestinal immunostimulation, as manifested by serum IgA, correlated with markers of inflammation, as manifested by the acute phase proteins, then gastrointestinal immunostimulation may play a part in the pathogenesis of inflammation in $\mathrm{AS}^{4}$ Moreover, one might expect that periods of increased AS inflammatory disease activity would be associated with heightened gastrointestinal immunostimulation, and this may 
possibly be reflected in increased concentrations of serum IgA.

Numerous investigators have studied the relation between disease activity, serum IgA, and serum CRP. Veys $e t$ al and Pease $e t$ al failed to show any association between serum IgA and disease activity in AS, ${ }^{17}{ }^{18}$ though Veys used a more restrictive definition of active disease (increased ESR or CRP). On the other hand, Laurent and Panayi showed that IgA concentrations were increased in active AS, though not significantly when compared with patients with inactive AS. ${ }^{19}$ Franssen et al showed a significant rise in serum IgA in active AS compared with inactive AS. ${ }^{20}$ Moreover, Ebringer et al showed that high titres of IgA against klebsiella were associated with active $\mathrm{AS}$, and that progression from inactive to active disease could be predicted by a rise in serum concentrations of $\operatorname{IgA}$ antibody to klebsiella. ${ }^{21}$

Contradictory results have been published on the association between disease activity and CRP concentrations. Pease et al could not show any association between CRP and clinically assessed disease activity. ${ }^{18}$ Laurent and Panayi, however, showed high concentrations of CRP in patients with AS with peripheral arthritis (extrapelvospinal), ${ }^{19}$ and Hussein et al showed that CRP is significantly associated with active disease in patients with juvenile spondyloarthropathies. ${ }^{22}$ Nashel et al suggested that CRP is both associated with active AS and is a better predictor of disease activity than ESR. ${ }^{23}$ Finally, when studying B27 + patients with AS Cowling et al showed that high concentrations of CRP correlated positively with both clinically assessed disease activity and positive klebsiella cultures in the stool, which again might implicate gastrointestinal immunostimulation as a trigger for AS.

As AS may be a heterogeneous group of phenotypically similar diseases ${ }^{12}{ }^{13}$ it is difficult to be certain that an observation on a reported sample of patients with AS is not skewed because of the varied proportion of diseases which merely phenotypically resemble each other. Therefore we studied randomly selected B27+ and B27- patients whose disease had been classified as either active or inactive to determine differences related to the presence or absence of B27.

We showed that the effects of B27 and the level of disease activity have a significant effect $(p<0.003)$ on the concentrations of serum IgA, $\mathrm{CRP}$, and haptoglobin. Moreover, further analysis showed that among the B27+ patients clinically active disease was associated with an increase in serum concentrations of both CRP $(p<0.017)$ and haptoglobin $(p<0.014)$; the concentration of serum IgA was not significantly altered. In contrast, in B27- patients disease activity was significantly associated only with serum IgA concentrations $(p<0.008)$; but not with concentrations of serum CRP or haptoglobin.

This evidence suggests different mechanisms for the pathogenesis of active inflammation in B27+ and B27- patients with AS. These results support the view that in patients who are B27+ active disease is associated with systemic inflammatory components manifested by a rise in the concentrations of the acute phase proteins. The increase in serum IgA seen in these patients may represent an early step in the pathogenic process and is consistent with the theory that gastrointestinal immunostimulation is a trigger that merely starts an inflammatory cascade. In comparison, although B27- patients with AS show a markedly increased concentration of serum $\operatorname{IgA}$, clinically inactive disease is associated with a higher concentration than is found in patients with active disease. The importance of this finding is difficult to decipher and needs confirmation by studying a larger group of B27- patients with AS. When we randomly selected a second group of B27- patients with AS from the original 303 Swiss patients we again found that clinically inactive disease was associated with higher concentrations of serum IgA than those found in patients with active disease.

This work emphasises the fact that as investigators have been able to study more B27patients they have been able to show clinical differences between B27+ and B27- AS. Several, epidemiological differences have been described between B27+ and B27- patients with AS: B27+ patients have a slightly earlier age of onset, often show familial aggregation, and develop acute anterior uveitis significantly more commonly than B27- patients. $^{21224}$ Now we have provided a demonstration of serological differences as well. This research further strengthens the possibility that AS represents a heterogeneous group of diseases and that the pathogenic mechanism in B27+ disease may differ from that in B27-AS. Our data thus add to the growing body of evidence supporting the clinical and genetic heterogeneity of AS.

Supported by the Irma Bender Arthritis Research Fund. We also gratefully acknowledge the technical assistance provided by Debra Schultz.

1 Goldin R H, Bluestone R. Tissue typing in rheumatic disease. Clin Rheum Dis 1976; 2: 231-52

2 Khan M A. Ankylosing spondylitis and heterogeneity of HLA-B27. Semin Arthritis Rheum 1988; 18: 134-41.

3 Woodrow J C. Genetic aspects of spondyloarthropathies. Clin Rheum Dis 1985; 1: 1-24.

4 Mackiewicz A, Khan M A, Reynolds T L, van der Linden S, Kushner I. Serum IgA, acute phase proteins, and glycosylation of alpha-1-acid glycoprotein in ankylosing spondylitis. Ann Rheum Dis 1989; 48: 99-103.

5 Trull A, Ebringer A, Panayi G, Ebringer R, James D C O. HLA-B27 and the immune response to enterobacterial antigens in ankylosing spondylitis. Clin Exp Immunol 1984; 55: 74-80.

6 Collado A, Sanmarti R, Brancos M A, et al. Immunoglobulin $A$ and $C$ reactive protein levels in ankylosing spondylitis. Ann Rheum Dis 1987; 46: 719-20.

7 Bennett P H, Burch T A. Population Studies of the rheumatic diseases. Amsterdam: Excerpta Medica, 1968: 456-7.

8 van der Linden S J, Valkenburg H A, Cats A. Evaluation of diagnostic criteria for ankylosing spondylitis: a proposal for diagnostic criteria for ankylosing spondylitis: a proposal for
modification of the New York criteria. Arthritis Rheum modification of the

9 Sheehan N J, Slavin B M, Donovan M P, Mount J N, Mathews J A. Lack of correlation between clinical disease activity and erythrocyte sedimentation rate, acute phase proteins or protease inhibitors in ankylosing spondylitis. $\mathrm{Br}$ f Rheumatol 1986; 25: 171-4.

10 Mancini G, Carbonara A O, Heremans J F. Immunochemical quantitation of antigens by single radial immunodiffusion. Immunochemistry 1965; 2: 361-8.

11 Mackiewicz A, Wiktorowicz K, Mackiewicz S. Comparison of three immunoassays for $\mathrm{C}$-reactive protein determination. Arch Immunol Ther Exp (Warsz) 1985; 33: 693-710.

12 Linssen A, Feltkamp T E W. B27 positive diseases versus B27 negative diseases. Ann R heum Dis 1988; 47: 431-9.

$13 \mathrm{Khan}$ M A. Newer clinical and radiographic features of seronegative spondyloarthropathies. Current Opinions in Rheumatology 1989; 1: 139-43. 
14 Cowling P, Ébringer R, Cawdell D, Ishii $M$, Ebringer $A$. $C$-reactive protein, ESR, and klebsiella in ankylosing spondylitis. Ann Rherum Dis 1980; 39: 45-9.

15 Schwimmbeck P L, Yu D T Y, Oldstone M B A Autoantibodies to HLA-B27 in the sera of HLA-B27 patients with ankylosing spondylitis (AS) and Reiter's syndrome: molecular mimicry with Klebsiella pneumoniae syndrome: molecular mimicry with Klebsiella pneumoniae as potential mechanis

16 Mielants H, Veys E M, eds. Ankylosing spondylitis and reactive arthritis, pathogenic aspects and therapeutic consequences. Ghent, Belgium: Omega Editions Medical Science Publishers, 1988.

17 Veys $E$ M, van Laere $M$. Serum IgG, IgM, and IgA levels in ankylosing spondylitis. Ann Rheum Dis 1973; 32: 493-6.

18 Pease C T, Fordham J N, Stubbs M M, Archer J R. IgA antibodies to gram-negative bacteria in the serum and saliva of patients with ankylosing spondylitis. Scand $\mathcal{f} R$ Reumatol 1987; 16: 199-203.

19 Laurent M R, Panayi G S. Acute-phase proteins and serum immunoglobulins in ankylosing spondylitis. Ann Rheum Dis 1983; 42: 524-8.

20 Franssen M J A M, van de Putte L B A, Gribnau F W J. IgA serum levels and disease activity in ankylosing spondylitis: a prospective study. Ann Rhewom Dis 1985; 44: 766-71.

21 Ebringer $R$ W, Cawdell D R, Cowling P, Ebringer A. Sequential studies in ankylosing spondylitis: association of 1978; 37: 146-51. 1978; 37: 146-51.

Hussein A, Stein J, Ehrich J H H. C-reactive protein in the assessment of disease activity in juvenile rheumatoid arthritis and juvenile spondyloarthritis. Scand $\mathcal{f}$ Rheumatol 1987; 16: 101-5.

23 Nashel D J, Petrone D L, Ulmer C C, Sliwinski A J. Creactive protein: a marker for disease activity in ankylosing spondylitis and Reiter's syndrome. $\mathcal{F}$ Rheumatol 1986; 13: 364-7.

24 Khan M A, Kusher I, Braun W E. Comparison of clinical features in HLA-B27 positive and negative patients with ankylosing spondylitis. Arthritis Rheum 1977; 20: 909-12. 